\title{
Differential Use of Screening Mammography in Older Women Initiating Metformin versus Sulfonylurea
}

\author{
Jin-Liern Hong ${ }^{1 *}$ (D), Louise M. Henderson ${ }^{1,2}$, Michele Jonsson Funk ${ }^{1}$, Jennifer L. Lund ${ }^{1}$, John B. Buse ${ }^{3}$, \\ Virginia Pate $^{1}$ and Til Stürmer ${ }^{1}$ \\ ${ }^{1}$ Department of Epidemiology, Gillings School of Global Public Health, University of North Carolina at Chapel Hill, Chapel Hill, NC, \\ USA \\ ${ }^{2}$ Department of Radiology, School of Medicine, University of North Carolina at Chapel Hill, Chapel Hill, NC, USA \\ ${ }^{3}$ Department of Medicine, School of Medicine, University of North Carolina at Chapel Hill, Chapel Hill, NC, USA
}

\begin{abstract}
Purpose Differential use of screening mammography may lead to biased detection of breast cancer. This study aimed to compare receipt of screening mammography and the incidence of screen-detected breast cancer between metformin and sulfonylurea initiators.

Methods We used 2006-2014 US Medicare claims to identify initiators of metformin or sulfonylurea aged 65+ years continuously enrolled in Parts $\mathrm{A} / \mathrm{B}$ for $\geq 2$ years pre-initiation and $\geq 2$ years post-initiation. We reported frequencies of screening mammograms and screen-detected breast cancer in 1 year pre-initiation among all cohort members and in 1 year post-initiation among cancer-free cohort members. Weighted screening risk differences (RDs) were estimated comparing metformin to sulfonylurea group.

Results We identified 41,436 and 13,367 initiators of metformin and sulfonylurea, $35 \%$ and $24 \%$ of which had $\geq 1$ screening mammogram in 1 year pre-initiation (weighted RD: 6 percentage points; 95\% CI: 5 to 7), respectively. The weighted RD for screen-detected breast cancer associated with metformin was 0.00 percentage points (95\% CI: -0.09 to 0.09 ). Among cancer-free cohort members, metformin initiators had 5 percentage points ( $95 \%$ CI: 4 to 6 ) and 0.11 percentage points (95\% CI: -0.02 to 0.23 ) absolute risk excess of screening mammography and screen-detected breast cancer in 1 year post-initiation, compared with sulfonylurea initiators, respectively.

Conclusions Metformin initiators were more likely to receive screening mammograms than sulfonylurea initiators pre- and post-initiation, indicating possible detection bias due to differential screening mammography. Researchers should be aware of the potential for more screening mammograms pre- and post-initiation when interpreting the findings of metformin on breast cancer incidence. Copyright $\left({ }^{\circ} 2017\right.$ John Wiley \& Sons, Ltd.
\end{abstract}

KEY WORDS-Diabetes; Metformin; Breast Cancer; Screening mammography

Received 6 August 2016; Revised 13 February 2017; Accepted 17 February 2017

\section{INTRODUCTION}

Metformin is the most commonly prescribed drug for type 2 diabetes. It has been linked with possible beneficial effects on breast cancer incidence in several observational studies ${ }^{1-4}$ but not in others. ${ }^{5-11}$ Many of the studies reporting an inverse association between metformin and breast cancer risk may suffer from

\footnotetext{
*Correspondence to: J.-L. Hong, Department of Epidemiology, Gillings School of Global Public Health, University of North Carolina at Chapel Hill, 135 Dauer Drive, McGavran Greenberg, Campus Box 7435, Chapel Hill, NC 27599-7435, USA. E-mail: jlhongtw@email.unc.edu

This article has not been published in a peer-reviewed journal, but a part of the results was previously presented at the 2015 annual meeting of the International Society for Pharmacoepidemiology.
}

time-related biases. ${ }^{12}$ Another potential source of bias, detection bias due to differential healthcare utilization, could also affect the metformin-breast cancer association but has not been addressed to date. Differential detection of asymptomatic or pre-clinical cancer before metformin initiation could reduce cancer incidence after initiation by excluding women whose breast cancer was detected at an early stage. On the other hand, differential detection after metformin initiation may lead to an increased incidence rate immediately following treatment initiation followed by a period of reduced incidence rate.

Our previous study examined healthcare utilization comparing metformin and a clinical alternative, sulfonylurea, using data from Medicare claims. ${ }^{13}$ We have noted that metformin initiators were more likely than 
sulfonylurea initiators to receive mammograms and to visit physicians around the time of initiation, potentially leading to biased detection of breast cancer. However, this study failed to distinguish screening from diagnostic mammography, thus the results did not reflect the actual difference in receipt of screening mammography between metformin and sulfonylurea initiators. Therefore, the objective of this study was to quantify the risk of screening mammography and screen-detected breast cancer over 12-month periods pre- and post-initiation among metformin and sulfonylurea initiators.

\section{METHODS}

\section{Study Population}

This study used data from Medicare fee-for-service beneficiaries from January $1^{\text {st }}, 2006$ to December $31^{\text {st }}, 2014$. Medicare offers free screening mammography annually for women with Medicare aged 40+, minimizing the influences of socioeconomic disparity. Given that differential detection pre- and postinitiation would affect breast cancer incidence estimates differently, this study included two study cohorts. One was the new user cohort which examined use of screening mammography over a two-year window (12 months pre- and post-initiation), primarily focusing on the period of 12 months before drug initiation. The second study cohort was the cancer-free cohort mimicking a cohort study on breast cancer incidence excluding individuals diagnosed with any cancer before drug initiation, and only examined use of screening mammography in 12 months after initiation.

For the new user cohort, we identified women aged 65 or older who initiated monotherapy of metformin or sulfonylurea between 2008 and 2012. Initiation was defined as having $\geq 1$ refill within 90 days after end of drug supply of the initial prescription and having $\geq 12$ month period of continuous Part D enrollment before initiation without use of any antihyperglycemic drugs. Patients were classified as initiators of metformin or sulfonylurea according to the initial prescription and the date of the first prescription was defined as the index date. Eligible patients were also required to be enrolled in Part A and B continuously for $\geq 24$ months pre-initiation and $\geq 24$ months post-initiation, thus patients who died or disenrolled Medicare Part A and B within 24 months after initiation were excluded from the study.

We utilized the cancer-free cohort to assess receipt of screening mammography within 12 months following treatment initiation among women with no history of cancer. This cancer-free cohort was a subgroup of the new user cohort, which included eligible initiators without a diagnosis of any cancer except for non-melanoma skin cancer within 12 months before initiation. The flowchart of the study cohort is available in Supplemental Figure S1.

\section{Screening Mammography}

We used the following Healthcare Common Procedure Coding System (HCPCS) codes to select all bilateral mammograms for the study cohort: G0202-G0205, 76091-76092, 77051-77052, and 77056-77057. Mammograms were further classified as either screening versus diagnostic using a claims-based algorithm ${ }^{14}$. Briefly, mammograms were considered as screening if they were coded as screening mammography without a previous mammogram within the prior 9 months and without a breast cancer diagnosis in the prior year. This algorithm has been validated in Medicare claims with a positive predictive value (PPV) of $94.9 \%^{14}$. Our application of this algorithm identified 36,164 (83\%) and 16,855 (91\%) screening mammograms out of 43,333 and 18,504 total mammograms in the new user cohort and the cancer-free cohort, respectively (Supplemental Figures S2-S3). Only screening mammograms were included in the analysis.

\section{Screen-Detected Breast Cancer}

After distinguishing screening from diagnostic mammograms, we used the Fenton algorithm to identify incident screen-detected breast cancers. ${ }^{15}$ This algorithm has a PPV of $88.0 \%$ among Medicare enrollees. ${ }^{15}$ The Fenton algorithm classifies screening mammograms as positive to detect breast cancer by requiring a breast cancer diagnosis within 123 days post-screening mammogram and a breast-directed surgery within a year following the diagnosis, or by a diagnosis of ductal carcinoma in-situ within 286 days post-screening mammogram with a subsequent mammogram within 82 days following the diagnosis. Our application of this algorithm and the results are shown in Supplemental Figures S4-S5. We further calculated the screening detection rate for breast cancer by dividing the number of screen-detected breast cancers by the number of screening mammograms.

\section{Incident Breast Cancer}

In the cancer-free cohort, any incident breast cancer during the 12-month follow-up was another outcome 
of interest, irrespective of whether it was detected by screening or due to symptoms. To be consistent with the Fenton algorithm, we required a breastdirected surgery within a year following a diagnosis code for invasive breast cancer and required a mammogram within 82 days after a diagnosis code for ductal carcinoma in-situ, to ascertain the breast cancer case.

\section{Statistical Analysis}

In the new user cohort, we calculated use of screening mammography in metformin and sulfonylurea initiators during a two-year window of 12 months pre- and post-initiation. The day of initiation was indexed as Month 0, and was included in the month following initiation (Month 1). Frequencies of initiators receiving a screening mammogram were summarized within sequential 3-month and 12-month intervals from Month -12 (before initiation) to Month 12 (after initiation), respectively. Incident breast cancer detected at screening was calculated over 12-monthly intervals, given expected small numbers. We estimated risks and risk differences (RDs) and their 95\% confidence intervals (CIs) of each event during each time interval comparing metformin to sulfonylurea initiators. To control the measured confounders, we used propensity score weighting methods to standardize sulfonylurea initiators to metformin initiators on age, race, comorbidities, calendar year of initiation, and number of physician office visit. ${ }^{16}$

In the cancer-free cohort, we repeated all analyses during the time window of 12 months after initiation only and calculated RD of the incidence of any breast cancer comparing metformin to sulfonylurea groups in 12 months post-initiation. Given that screening mammography before initiation is likely associated with receiving subsequent screening tests after initiation, we additionally included the variable of prior screening in the propensity score model for confounding control. Furthermore, the analyses in the cancer-free cohort were stratified by receipt of screening mammography in 12 months before initiation.

All analyses are performed with the SAS software v9.3 (Cary, NC).

\section{RESULTS}

Our new user cohort included 41,436 initiators of metformin and 13,367 initiators of sulfonylurea during the study period. Table 1 summarizes the baseline characteristics and the proportion of women who had $\geq 1$ screening mammogram in 2 years between metformin and sulfonylurea initiators, respectively. Over a two-year window, $47 \%$ of the new user cohort received $\geq 1$ screening mammogram. We identified 29,597 and 6,567 screening mammograms over two years in $50 \%$ of metformin initiators and $36 \%$ of sulfonylurea initiators, respectively. The proportion of women receiving screening mammograms decreased with age but increased with number of physician visits in both groups. Screening mammography was less common in the patients with comorbidity, such as those with cardiovascular disease or hospital admission, but remained similar across calendar years.

We present the proportion of patients receiving screening mammograms in 3-monthly intervals over 12 months before and after initiation in Figure 1. Metformin initiators were more likely to have screening mammograms than sulfonylurea initiators, which was consistent over time. For both cohorts, the percentage of patients receiving screening mammograms peaked in the 3 months immediately following initiation in metformin initiators, but was fairly stable over time.

Comparing metformin to sulfonylurea initiators, the weighted RD of screening mammography over the two-year window was 8 percentage points $(95 \% \mathrm{CI}$ : 7 to 9) (Table 2). The results were similar before and after initiation. We identified 197 and 37 incident breast cancers that were detected on screening mammograms among metformin and sulfonylurea groups, respectively (Table 2). The risk of incident screendetected breast cancer was $0.48 \%$ in metformin, as compared with $0.28 \%$ in sulfonylurea, with a weighted RD of 0.11 percentage points (95\% CI: -0.03 to 0.25 ). A higher absolute risk of screen-detected breast cancer was observed for the metformin group in the 12 months after initiation (weighted RD: 0.11 percentage points; $95 \%$ CI: 0.00 to 0.21 ), but not during the 12 months before initiation.

In the cancer-free cohort, we observed similar patient characteristics and patterns of screening mammography and screen-detected breast cancer during 12 months after initiation in the cancer-free cohort (Supplemental Table S2 and Table 3). Metformin initiators in the cancer-free cohort were also more likely to receive screening mammograms than sulfonylurea initiators (weighted RD: 5 percentage points; 95\% CI: 4 to 6). The risk of screendetected breast cancer was higher in metformin $(0.32 \%)$ than sulfonylurea $(0.13 \%)$, but differences became attenuated after accounting for imbalances in baseline characteristics (weighted RD: 0.11 percentage 
Table 1. Characteristics at baseline among metformin and sulfonylurea initiators in the new user cohort, and proportion of women receiving at least one screening test within a 2-year window of 12 months before and after initiation.

\begin{tabular}{|c|c|c|c|c|c|c|c|}
\hline \multirow[b]{2}{*}{ Characteristics } & \multicolumn{3}{|c|}{ Metformin } & \multicolumn{3}{|c|}{ Sulfonylurea } & \multirow{2}{*}{$\begin{array}{c}\text { Screening } \\
\text { Difference } \\
(\%)^{\dagger}\end{array}$} \\
\hline & \multicolumn{2}{|c|}{$\mathrm{N}(\%)$} & \multirow{2}{*}{$\frac{\% \text { screened* }}{50.3}$} & \multicolumn{2}{|c|}{$\mathrm{N}(\%)$} & \multirow{2}{*}{$\begin{array}{c}\text { \% screened* } \\
35.7\end{array}$} & \\
\hline Total No. & 41,436 & $(100.0)$ & & 13,367 & $(100.0)$ & & 14.6 \\
\hline Age, years & \multirow{3}{*}{\multicolumn{2}{|c|}{$\begin{array}{c}73 \\
(69-78)\end{array}$}} & & \multirow{3}{*}{\multicolumn{2}{|c|}{$\begin{array}{c}76 \\
(70-83)\end{array}$}} & & \\
\hline Median & & & $72^{\S}$ & & & $74^{\S}$ & \\
\hline Interquartile Range (IQR) & & & $(69-77)$ & & & $(69-80)$ & \\
\hline \multicolumn{8}{|l|}{ Category } \\
\hline $65-69$ & 12,256 & $(29.6)$ & 54.0 & 3,077 & $(23.0)$ & 39.1 & 15.0 \\
\hline $70-74$ & 12,159 & $(29.3)$ & 57.7 & 2,609 & $(19.5)$ & 47.9 & 9.8 \\
\hline $75-79$ & 8,115 & $(19.6)$ & 51.9 & 2,549 & $(19.1)$ & 41.0 & 10.9 \\
\hline $80-84$ & 5,295 & $(12.8)$ & 41.0 & 2,442 & $(18.3)$ & 33.2 & 7.8 \\
\hline $85+$ & 3,611 & $(8.7)$ & 22.9 & 2,690 & $(20.1)$ & 17.1 & 5.8 \\
\hline \multicolumn{8}{|l|}{ Race } \\
\hline White & 33,447 & $(80.7)$ & 51.4 & 10,468 & $(78.3)$ & 36.2 & 15.2 \\
\hline Black & 4,237 & $(10.2)$ & 49.5 & 1,823 & $(13.6)$ & 37.4 & 12.1 \\
\hline Others & 3,752 & $(9.1)$ & 41.8 & 1,076 & $(8.0)$ & 27.7 & 14.1 \\
\hline \multicolumn{8}{|l|}{ Comorbidity $^{\mathrm{II}}$} \\
\hline Breast Cancer & 2,361 & $(5.7)$ & 9.7 & 715 & $(5.3)$ & 7.7 & 2.0 \\
\hline Any Cancer & 5,610 & $(13.5)$ & 35.7 & 2,008 & $(15.0)$ & 29.2 & 6.4 \\
\hline Congestive Heart Failure & 5,066 & $(12.2)$ & 35.6 & 3,187 & $(23.8)$ & 26.5 & 9.2 \\
\hline Ischemic Heart Disease & 9,992 & $(24.1)$ & 45.5 & 4,521 & $(33.8)$ & 33.7 & 11.8 \\
\hline Hypertension & 34,737 & $(83.8)$ & 50.9 & 11,412 & $(85.4)$ & 36.4 & 14.5 \\
\hline \multicolumn{8}{|l|}{ HealthCare Utilization } \\
\hline \multicolumn{8}{|l|}{ Days of Hospitalization ${ }^{\text {I }}$} \\
\hline 0 & 33,653 & $(81.2)$ & 52.6 & 9,173 & $(68.6)$ & 38.9 & 13.8 \\
\hline 1 to 7 & 5,364 & $(12.9)$ & 44.0 & 2,481 & $(18.6)$ & 30.4 & 13.6 \\
\hline $8+$ & 2,419 & $(5.8)$ & 31.9 & 1,713 & $(12.8)$ & 26.0 & 5.9 \\
\hline \multicolumn{8}{|l|}{ No. of Physician Office Visit ${ }^{\mathrm{gl}}$} \\
\hline$\leq 3$ & 6,184 & $(14.9)$ & 31.8 & 2,467 & $(18.5)$ & 19.4 & 12.4 \\
\hline 4 to 6 & 4,971 & $(12.0)$ & 41.9 & 1,419 & $(10.6)$ & 26.3 & 15.7 \\
\hline 7 to 12 & 10,303 & $(24.9)$ & 52.1 & 2,973 & $(22.2)$ & 35.0 & 17.1 \\
\hline $13+$ & 19,978 & $(48.2)$ & 57.2 & 6,508 & $(48.7)$ & 44.2 & 13.0 \\
\hline \multicolumn{8}{|l|}{ Location } \\
\hline Mid West & 10,522 & $(25.4)$ & 52.8 & 3,441 & $(25.7)$ & 37.2 & 15.6 \\
\hline North East & 6,655 & $(16.1)$ & 49.6 & 2,467 & $(18.5)$ & 34.4 & 15.2 \\
\hline South & 16,521 & $(39.9)$ & 49.8 & 5,583 & $(41.8)$ & 35.5 & 14.3 \\
\hline West & 7,509 & $(18.1)$ & 48.8 & 1,786 & (13.4) & 35.6 & 13.3 \\
\hline Others & 220 & $(0.5)$ & 38.2 & 82 & $(0.6)$ & 22.0 & 16.2 \\
\hline \multicolumn{8}{|l|}{ Calendar Year of Initiation } \\
\hline 2008 & 8,180 & $(19.7)$ & 51.2 & 3,262 & $(24.4)$ & 34.9 & 16.3 \\
\hline 2009 & 8,365 & $(20.2)$ & 51.0 & 2,993 & $(22.4)$ & 36.0 & 15.0 \\
\hline 2010 & 8,304 & $(20.0)$ & 50.5 & 2,556 & $(19.1)$ & 34.5 & 16.0 \\
\hline 2011 & 8,310 & $(20.1)$ & 49.7 & 2,317 & $(17.3)$ & 36.2 & 13.5 \\
\hline 2012 & 8,277 & $(20.0)$ & 49.1 & 2,239 & $(16.8)$ & 37.2 & 12.0 \\
\hline
\end{tabular}

$* \%$ screened was calculated as proportion of women receiving $\geq 1$ screening mammogram within 2-year window of 12 months before and after initiation among the initiators. We also calculated the percentage of women screened in 1 year before and after initiation, respectively. The results are presented in Supplemental Table S1.

${ }^{\dagger}$ Screening Difference (\%) was calculated as the difference in the proportion of women receiving $\geq 1$ screening mammogram within a 2 -year window of 12 months before and after initiation between initiators of metformin and sulfonylurea.

${ }^{\S}$ The median (IQR) of age was calculated for women receiving $\geq 1$ screening mammogram within 2-year window of 12 months before and after initiation for each drug cohort.

${ }^{\mathrm{IT}}$ Comorbidity was defined as the presence of one diagnosis code within 1 year prior to initiation. Days of hospitalization and number (no.) of physician office visits were defined within 1 year prior to initiation.

points; $95 \%$ CI: -0.02 to 0.23 ). For any incident breast cancer within 1 year post-initiation, metformin was not associated with an altered risk (weighted RD: 0.09 percentage points; $95 \% \mathrm{CI}$ : -0.07 to 0.26 ); however, more breast cancer cases were detected by screening in metformin initiators $(61 \%)$ than sulfonylurea initiators $(37 \%)$.
In the cancer-free cohort, we stratified the analyses by receipt of screening mammography in the 12 months before initiation (Table 3). Women screened in the 12 months pre-initiation were more likely to receive screening mammograms in the 12 months after initiation compared to those not screened before initiation, in both metformin and sulfonylurea groups. 


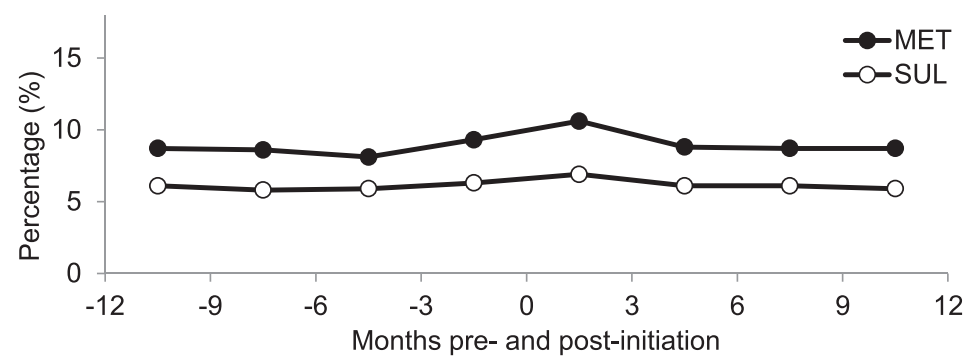

\begin{tabular}{lllllllll}
\multicolumn{2}{l}{ Proportion of patients who had screening mammograms } \\
MET, \% & 8.7 & 8.6 & 8.1 & 9.3 & 10.6 & 8.8 & 8.7 & 8.7 \\
SUL, \% & 6.1 & 5.8 & 5.9 & 6.3 & 6.9 & 6.1 & 6.1 & 5.9 \\
Difference, \% & 2.6 & 2.8 & 2.2 & 2.9 & 3.7 & 2.7 & 2.6 & 2.8
\end{tabular}

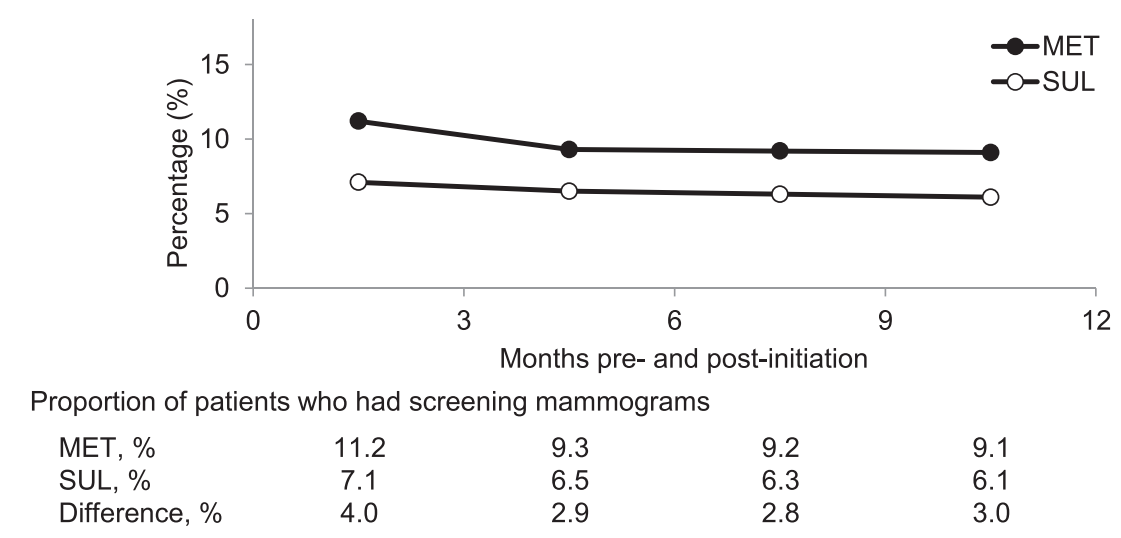

Figure 1. Proportions of women receiving a screening mammogram over time by treatment group, in the new user cohort (Top) and in the cancer-free cohort (Bottom).

The positive association between metformin and higher risks of screen-detected and all breast cancer was found among women not screened pre-initiation, but not among women previously screened.

\section{DISCUSSION}

In this study, we found that older women initiating metformin were not only more likely to be screened for breast cancer in 12 months before initiation than women initiating sulfonylurea, but they were also more likely to have screening mammograms in the 12 months after initiation. Consequently, compared with sulfonylurea initiators, metformin initiators had higher probabilities of screen-detected breast cancer, particularly after initiation. These results indicate existence of detection bias due to differential screening mammography pre- and post-initiation when comparing breast cancer incidence between women initiating metformin vs sulfonylurea.

A total of $47 \%$ of all initiators in our study underwent $\geq 1$ screening mammogram over the two-year time window, far less than that reported by the U.S. Centers for Disease Control and Prevention
(CDC). The CDC estimated $65.5 \%$ of women aged $65+$ years in 2008 self-reported receiving a mammogram within the past 2 years based on the National Health Interview Survey ${ }^{17}$, but the self-reported rates of cancer screenings tend to be overestimated. ${ }^{18,19} \mathrm{~A}$ previous study using the 5\% Medicare random sample found similar results to ours, which reported $40.2 \%$ of women aged 65+ years in Medicare during 2005-2006 had $\geq 1$ screening mammography. ${ }^{20} \mathrm{We}$ also observed that receipt of screening mammography was associated inversely with age but positively with number of physician visits, consistent with previous findings. ${ }^{20}$ The United States Preventive Services Task Force (USPSTF) recommended yearly screening mammography for women aged $40+$ since $2002^{21}$, but revised the guideline to recommend biennial mammography for women aged 50-74 years in 2009. ${ }^{22}$ Our study found no difference in use of screening mammography in women aged 65+ years across calendar years, similar to previous national survey-based studies. ${ }^{17,23}$

Differential use of screening mammography between metformin and sulfonylurea initiators could be partially explained by age. Older women are less likely to receive screening mammography (Table 1) 
Table 2. Frequency of screening mammograms and screen-detected breast cancer over 2 years in the new user cohort, by treatment group and by time interval before and after initiation

\begin{tabular}{|c|c|c|c|c|c|c|}
\hline \multirow[b]{2}{*}{ Clinical Event } & & & \multicolumn{4}{|c|}{ Screening mammograms before and after initiation } \\
\hline & \multicolumn{2}{|c|}{ Mammograms over 24 months } & \multicolumn{2}{|c|}{12 months before initiation } & \multicolumn{2}{|c|}{12 months after initiation } \\
\hline Cohort & MET & SUL & MET & SUL & MET & SUL $^{\#}$ \\
\hline Total Patients, $\mathrm{n}$ & 41,436 & 13,367 & 41,436 & 13,367 & 41,436 & 13,367 \\
\hline \multicolumn{7}{|l|}{ Screening Mammography ${ }^{\text {II }}$} \\
\hline $\mathrm{N}$ of screening mammograms & 29,597 & 6,567 & 14,347 & 3,226 & 15,250 & 3,341 \\
\hline $\begin{array}{l}\mathrm{N} \text { of patients receiving } \geq 1 \text { screening } \\
\text { mammogram, } \mathrm{n}(\%)\end{array}$ & $20,847(50.3)$ & $4,767(35.7)$ & $14,328(34.6)$ & $3,223(24.1)$ & $15,223(36.7)$ & $3,337(25.0)$ \\
\hline Crude RD, $\%(95 \% \mathrm{CI})^{*}$ & \multicolumn{2}{|c|}{$14.6(13.7,15.6)$} & \multicolumn{2}{|c|}{$10.5(9.6,11.3)$} & \multicolumn{2}{|c|}{$11.8(10.9,12.6)$} \\
\hline Weighted RD,\% $(95 \% \mathrm{CI})^{* \dagger}$ & \multicolumn{2}{|c|}{$8.4(7.4,9.5)$} & \multicolumn{2}{|c|}{$6.4(5.4,7.4)$} & \multicolumn{2}{|c|}{$6.8(5.8,7.8)$} \\
\hline \multicolumn{7}{|l|}{ Breast Cancer detected at Screening } \\
\hline Screen-detected BC case, $\mathrm{n}(\%)$ & $197(0.48)$ & $37(0.28)$ & $73(0.18)$ & $20(0.15)$ & $124(0.30)$ & $17(0.13)$ \\
\hline Crude RD, \% (95\% CI)* & \multicolumn{2}{|c|}{$0.20(0.09,0.31)$} & \multicolumn{2}{|c|}{$0.03(-0.05,0.10)$} & \multicolumn{2}{|c|}{$0.17(0.09,0.25)$} \\
\hline Weighted RD,\% $(95 \% \mathrm{CI})^{* \dagger}$ & \multicolumn{2}{|c|}{$0.11(-0.03,0.25)$} & \multicolumn{2}{|c|}{$0.00(-0.09,0.09)$} & \multicolumn{2}{|c|}{$0.11(0.00,0.21)$} \\
\hline $\begin{array}{l}\text { Screening detection rate for breast } \\
\text { cancer per } 1,000 \text { tests }^{\S}\end{array}$ & $6.7(5.7,7.6)$ & $5.6(3.8,7.4)$ & $5.1(3.9,6.3)$ & $6.2(3.5,8.9)$ & $8.1(6.7,9.6)$ & $5.1(2.7,7.5)$ \\
\hline
\end{tabular}

Abbreviations: BC: breast cancer; N: number; MET: metformin; SUL: sulfonylurea; RD: risk difference.

*RD was estimated comparing metformin initiators to sulfonylurea initiators.

${ }^{\dagger}$ Weighted by standardizing to their distribution in metformin initiators by using weights of 1 for metformin initiators and the odds of the estimated propensity score for sulfonylurea initiators. Propensity score model included age (in years), age-squared, race category (white, black, and others), congestive heart failure (Yes/No), ischemic heart disease (Yes/No), hypertension (Yes/No), cancer (Yes/No), calendar year of initiation, and number of physician visit in 12 months prior to initiation (classified as $\leq 6,7$ to 12 , and $\geq 13$ ).

${ }^{\S}$ Screening detection rate for breast cancer was calculated by total cases of screen-detected breast cancer divided by total number of screening mammograms. The overall detection rate for screen-detected breast cancer was 6.5 per 1,000 screening examinations in our study, similar to the Breast Cancer Surveillance Consortium 2009 data which ranges from 6.1 to 8.5 as age increases from 65 to $85+.7$

${ }^{*}$ We conducted a sensitivity analysis in which a surgery was not required for a breast cancer diagnosis to identify incident breast cancer detected at screening mammography (Supplemental Table S4). The risk of screen-detected breast cancer between metformin and sulfonylurea initiators over 24 months was 0.22 percentage points $(95 \%$ CI: $0.04,0.40)$ after propensity score adjustment.

"We compared the performance of the algorithms between women aged $<75$ years and women aged 75 or older (Supplemental Table S6) and found no difference. The percentage of screening mammograms identified from all mammograms was $84 \%$ in women aged $<75$ and $82 \%$ in women aged 75 or older. The screening detection rate for breast cancer per 1,000 tests was 6.6 and 6.3 in women aged $<75$ or $75+$ years, respectively.

${ }^{\#}$ We examined the impact of subsequent addition of metformin on use of screening mammograms by stratifying sulfonylurea initiators based on subsequent addition of metformin in 12 months after initiation (Supplemental Table S7). 29\% and 24\% of sulfonylurea initiators adding and not adding metformin later received screening mammograms in 12 months, respectively, which were still lower than metformin initiators (37\%).

because mammography is recommended for women aged 50-74. Sulfonylurea initiators were older than metformin initiators, thus resulting in a lower proportion of women being screened. Nevertheless, differential screening between metformin and sulfonylurea initiators remains apparent after we controlled imbalance in age and patient characteristics by propensity score weighting. We hypothesize that it could also be related to prescriber's behavior. Previous studies have shown that physician recommendation is a strong motivation for undergoing screening mammography. ${ }^{24,25}$ Given that metformin is recommended as the preferred initial treatment for diabetes, metformin prescribers who comply with guideline recommendations may be more likely to perform regular examinations or to recommend cancer screening tests for older patients. Thus, women initiating metformin may be more likely to receive screening mammography and, consequently, to be diagnosed with breast cancer around the time of initiation. Besides, there is a possibility that use of metformin or sulfonylurea itself might alter the probability of being screened.
Metformin has a beneficial effect on weight loss which may lead to more frequent or better detection. Sulfonylurea users may receive more medical attention due to severe adverse effect such as hypoglycemia and this may detract from on cancer screenings.

Differential detection of breast cancer due to differential screening mammography before and after drug initiation has distinct influences on effect estimates, thus our results need to be interpreted separately. Differential screening mammography before initiation which we observed in the new user cohort suggests that metformin initiators may be at slightly lower risk of breast cancer than sulfonylurea initiators at the time of initiation because more women with asymptomatic breast cancer were excluded from the metformin group, despite the fact that the difference in the probability of screen-detected breast cancer was small.

On the other hand, differential screening mammography after initiation may increase breast cancer incidence in metformin initiators immediately following treatment initiation and may decrease breast cancer incidence thereafter because of the detection of 
Table 3. Frequency of screening mammograms, screen-detected breast cancer, and any breast cancer over 12 months after initiation in the cancer-free cohort, by treatment group and by receipt of screening mammography within 12 months prior to initiation.

\begin{tabular}{|c|c|c|c|c|c|c|}
\hline \multirow[b]{2}{*}{ Clinical Event } & \multirow{2}{*}{\multicolumn{2}{|c|}{$\begin{array}{c}\text { Mammograms in } 12 \text { months after } \\
\text { initiation }\end{array}$}} & \multicolumn{4}{|c|}{ Receipt of screening mammography within 12 months prior to initiation } \\
\hline & & & \multicolumn{2}{|c|}{ Yes } & \multicolumn{2}{|c|}{ No } \\
\hline Cohort & MET & SUL** & MET & SUL & MET & SUL \\
\hline Total Patients, $\mathrm{n}$ & 35,826 & 11,359 & 13,007 & 2,808 & 22,819 & 8,551 \\
\hline \multicolumn{7}{|l|}{ Screening Mammography \& } \\
\hline $\mathrm{N}$ of screening mammograms & 13,894 & 2,961 & 8,040 & 1,587 & 5,854 & 1,374 \\
\hline $\begin{array}{l}\mathrm{N} \text { of patients receiving } \geq 1 \\
\text { screening mammogram, } \mathrm{n}(\%)\end{array}$ & $13,868(38.7)$ & $2,957(26.0)$ & $8,028(61.7)$ & $1,585(56.4)$ & $5,840(25.6)$ & $1,372(16.0)$ \\
\hline Crude RD, \% (95\% CI)* & \multicolumn{2}{|c|}{$12.7(11.7,13.6)$} & \multicolumn{2}{|c|}{$5.3(3.3,7.3)$} & \multicolumn{2}{|c|}{$9.5(8.6,10.5)$} \\
\hline Weighted RD, $\%(95 \% \mathrm{CI})^{*}, \dagger$ & \multicolumn{2}{|c|}{$5.4(4.2,6.5)$} & \multicolumn{2}{|c|}{$4.2(2.0,6.4)$} & \multicolumn{2}{|c|}{$6.0(4.8,7.1)$} \\
\hline \multicolumn{7}{|l|}{ Breast Cancer detected at Screening } \\
\hline Screen-detected BC case, n (\%) & $114(0.32)$ & $15(0.13)$ & $42(0.32)$ & $<11(<0.39)^{\mathrm{q}}$ & $72(0.32)$ & $<11(<0.13)$ qा \\
\hline Crude RD, $\%(95 \% \mathrm{CI})^{*}$ & \multicolumn{2}{|c|}{$0.19(0.10,0.27)$} & \multicolumn{2}{|c|}{$\mathrm{N} / \mathrm{S}^{\mathrm{II}}$} & \multicolumn{2}{|c|}{$\mathrm{N} / \mathrm{S}^{\mathrm{qI}}$} \\
\hline Weighted RD,\% (95\% CI)* ${ }^{\dagger}$ & \multicolumn{2}{|c|}{$0.11(-0.02,0.23)$} & \multicolumn{2}{|c|}{$-0.06(-0.35,0.23)$} & \multicolumn{2}{|c|}{$0.20(0.08,0.31)$} \\
\hline $\begin{array}{l}\text { Screening detection rate for breast } \\
\text { cancer per } 1,000 \text { tests }^{\S}\end{array}$ & $8.2(6.7,9.7)$ & $5.1(2.5,7.6)$ & $5.2(3.6,6.8)$ & $\mathrm{N} / \mathrm{S}^{\mathrm{II}}$ & $12.3(9.5,15.1)$ & $\mathrm{N} / \mathrm{S}^{\mathrm{q}}$ \\
\hline \multicolumn{7}{|l|}{ Any Incident Breast Cancer } \\
\hline $\mathrm{BC}$ cases $(\%)$ & $186(0.52)$ & $41(0.36)$ & $58(0.45)$ & $14(0.50)$ & $128(0.56)$ & $27(0.32)$ \\
\hline Crude RD, $\%(95 \% \mathrm{CI})^{*}$ & \multicolumn{2}{|c|}{$0.16(0.03,0.29)$} & \multicolumn{2}{|c|}{$-0.05(-0.34,0.23)$} & \multicolumn{2}{|c|}{$0.25(0.09,0.40)$} \\
\hline Weighted RD,\% (95\% CI)* & \multicolumn{2}{|c|}{$0.09(-0.07,0.26)$} & \multicolumn{2}{|c|}{$-0.15(-0.50,0.20)$} & \multicolumn{2}{|c|}{$0.23(0.06,0.40)$} \\
\hline$\%$ of $\mathrm{BC}$ detected by screening ${ }^{\ddagger}$ & $61.3(54.3,68.3)$ & $36.6(21.8,51.3)$ & $72.4(60.9,83.9)$ & $\mathrm{N} / \mathrm{S}^{\mathrm{TI}}$ & $56.3(47.7,64.8)$ & $\mathrm{N} / \mathrm{S}^{\mathrm{qI}}$ \\
\hline
\end{tabular}

Abbreviations: BC: breast cancer; N: number; MET: metformin; SUL: sulfonylurea; RD: risk difference; N/S: not specified.

*RD was estimated comparing metformin initiators to sulfonylurea initiators.

Weighted by standardizing to their distribution in metformin initiators by using weights of 1 for metformin initiators and the odds of the estimated propensity score for sulfonylurea initiators. Propensity score model included age (in years), age-squared, race category (white, black, and others), congestive heart failure (Yes/No), ischemic heart disease (Yes/No), hypertension (Yes/No), cancer (Yes/No), calendar year of initiation, and number of physician visit in 12 months prior to initiation (classified as $\leq 6,7$ to 12 , and $\geq 13$ )

${ }^{\S}$ Screening detection rate for breast cancer was calculated by total cases of screen-detected breast cancer divided by total number of screening mammograms.

${ }^{\ddagger}$ Percent of breast cancer detected by screening was calculated by cases of screen-detected breast cancer divided by total cases of breast cancers

"I Our DUA does not allow us to present cell sizes $<11$, so the number for these cells were not presented on this table.

${ }^{\#}$ We conducted a sensitivity analysis in which a surgery was not required for a breast cancer diagnosis to identify incident breast cancer detected at screening mammography (Supplemental Table S5). The risk of screen-detected breast cancer between metformin and sulfonylurea initiators was 0.18 percentage points (95\% CI: $0.04,0.32)$ after propensity score adjustment.

${ }^{\&}$ We compared the performance of the algorithms between women aged $<75$ years and women aged 75 or older (Supplemental Table S6) and found no difference. The percentage of screening mammograms identified from all mammograms was $91 \%$ in women aged $<75$ and $97 \%$ in women aged 75 or older. The screening detection rate for breast cancer per 1,000 tests was 7.6 and 7.8 in women aged $<75$ or $75+$ years, respectively.

**We examined the impact of subsequent addition of metformin on use of screening mammograms by stratifying sulfonylurea initiators based on subsequent addition of metformin in 12 months after initiation (Supplemental Table S8). 30\% and 25\% of sulfonylurea initiators adding and not adding metformin later received screening mammograms in 12 months, respectively, which were still lower than metformin initiators (39\%).

preclinical breast cancer that may eventually become clinical and be diagnosed without screening. One cohort study examined metformin users in the UK Clinical Practice Research Datalink (CPRD) and found a pattern of higher breast cancer risk in the first 6 months since initiation compared with later ${ }^{4}$, supporting our hypothesis. This may also explain the unexpected increased incidence of breast cancer in metformin users which was observed in one cohort study of US Medicare claims ${ }^{10}$, and, in addition to time-related bias ${ }^{12}$, might also partially explain the benefits of metformin on breast cancer incidence which was only observed after long-term treatment in one CPRD case-control study. ${ }^{1}$

Cohort studies of cancer incidence usually consider a lag period of 6 or 12 months and follow-up begins after the lag period. The lag period is important because it explicitly incorporates into the analysis a time-window for the cancer to develop and be detected. On the other hand, differential screening after initiation could lead to a relative lower risk of breast cancer for metformin initiators given more cases of screen-detected breast cancer are excluded from metformin initiators than sulfonylurea initiators during the lag period. Together, our results on differential screening mammography are unlikely to explain why we would observe a null association between metformin in comparison with sulfonylurea and breast cancer as observed in some studies assuming a real protective effect of metformin. ${ }^{5,8,9}$ Our results might, however, partially explain a reduced risk of breast cancer associated with metformin as observed in one cohort study with sulfonylurea as a comparison group ${ }^{3}$ and in several studies with different comparison groups $s^{1,2,7}$ assuming no effect of metformin on breast cancer incidence. 
In the cancer-free cohort, increased risks of screendetected breast cancer and all breast cancer in metformin initiators were confined to women not screened previously. Differential detection has little impact on the association between metformin and breast cancer incidence among women previously screened. The findings suggest that restricting the study population to women with prior screening may be effective to reduce detection bias due to differential use of screening mammography. In addition, estimating the proportion of study population receiving screening mammograms before and after initiation can be useful to quantify the impact of differential detection.

This study has some limitations. First, our study is limited by the small number of screen-detected breast cancer cases, thus the effect estimates (i.e., RD) were imprecise. We acknowledge that this impacted our ability to detect a small difference in the risk of screen-detected breast cancer during the study period. Secondly, we may have underestimated true cases of breast cancer detected at screening. To ascertain breast cancer cases, we required a breast surgery following a breast cancer diagnosis, according to the Fenton algorithm. ${ }^{14}$ Although surgery is the primary and most effective treatment for breast cancer, it is possible that older women with comorbid conditions may not undergo surgery. ${ }^{26}$ Thirdly, there might be residual confounding by age. After propensity score adjustment, metformin and sulfonylurea initiators were marginally comparable in age. Imbalance in age may still remain among extremely old patients with comorbidities, but is expected to be small. In addition, given the limited sample size, we did not use information on prescriber's characteristics to evaluate our hypothesis that differential screening may be related to prescriber's behavior. Although the algorithms used in this study been validated within Medicare claims, we acknowledge the difficulty to distinguish true screening from diagnostic mammography because women with symptoms may be more likely to undergo screening. Lastly, Medicare part B plans provide free annual screening mammography for women aged 65 + , reducing health inequalities for receiving mammography. Thus, our results can be generalized to US older women, but may not be generalized to younger women or women residing in other countries where socioeconomic status likely affects the probability of receiving screening mammography.

Our study provides empirical evidence for biased detection for breast cancer due to more screening mammograms being performed in older women initiating metformin compared with sulfonylurea around the time of drug initiation. Researchers should be aware of the potential for more screening mammograms pre- and post-initiation when interpreting the findings of studies assessing the effects of metformin on breast cancer incidence.

\section{DISCLOSURE OF POTENTIAL CONFLICTS OF INTEREST}

TS receives investigator-initiated research funding and support as Principal Investigator (R01 AG023178) from the National Institute on Aging (NIA), and as Co-Investigator (R01 CA174453; R01 HL118255, R21-HD080214), National Institutes of Health (NIH). $\mathrm{He}$ also receives salary support as Director of the Comparative Effectiveness Research (CER) Strategic Initiative, NC TraCS Institute, UNC Clinical and Translational Science Award (UL1TR001111) and as Director of the Center for Pharmacoepidemiology (current members: GlaxoSmithKline, UCB BioSciences, Merck) and research support from pharmaceutical companies (Amgen, AstraZeneca) to the Department of Epidemiology, University of North Carolina at Chapel Hill. Dr. Stürmer does not accept personal compensation of any kind from any pharmaceutical company. He owns stock in Novartis, Roche, BASF, AstraZeneca, and Johnsen \& Johnsen.

MJF receives investigator-initiated research funding and support as Principal Investigator from the National Institutes of Health (NIH), National Heart Lung and Blood Institute (NHLBI, R01 HL118255); as a Co-Investigator on grant awards from the $\mathrm{NIH}$ National Institute on Aging (NIA, R01 AG023178), the NIH National Center for Advancing Translational Sciences (NCATS, 1UL1TR001111), and AstraZeneca. Dr. Jonsson Funk does not accept personal compensation of any kind from any pharmaceutical company, though she receives salary support from the Center for Pharmacoepidemiology in the Department of Epidemiology, Gillings School of Global Public Health (current members: GlaxoSmithKline, UCB BioSciences, Merck).

JB is an investigator, consultant, or both (without any direct financial benefit) under contracts between UNC-CH and Andromeda, AstraZeneca, Bayhill Therapeutics, Boehringer Ingelheim, Bristol-Myers Squibb, Elcelyx Therapeutics, Eli Lilly, GI Dynamics, GlaxoSmithKline, Halozyme Therapeutics, F Hoffmann-La Roche, Intarcia Therapeutics, Johnson \& Johnson, Lexicon, LipoScience, Medtronic, Merck, Metabolon, Metavention, Novo Nordisk, Orexigen Therapeutics, Osiris Therapeutics, Pfizer, Quest Diagnostics, Rhythm Pharmaceuticals, Sanofi, Takeda, ToleRx, TransTech Pharma, Veritas, and 
Verva. He is a consultant to PhaseBio and is personally in receipt of stock options for that work.

LMH receives research funding from NIH/NCI (R01CA155342, R21CA175983, P01CA154292, HHSN261201100031C, R01CA149365) and PCORI (Comparative effectiveness of surveillance modalities breast cancer survivors). LMH does not accept personal compensation of any kind from any pharmaceutical company.

JLL receives research funding from the UNC Oncology Clinical Translational Research Training Program (5K12CA120780) and as the Principal Investigator of a Research Starter Award from the Pharmaceutical Research and Manufacturers of America (PhRMA) Foundation. Dr. Lund does not accept personal compensation of any kind from any pharmaceutical company.

No potential conflicts of interest were disclosed by the other authors.

\section{KEY POINTS}

- In the US, older women initiating metformin are more likely than those initiating sulfonylurea to be screened for breast cancer in the year before and after initiation.

- More screening mammography pre-initiation would tend to reduce breast cancer incidence in metformin initiators; in contrast, more screening mammography post-initiation would tend to increase breast cancer incidence in metformin initiators.

- Researchers should be aware of the potential for more screening mammograms in women initiating metformin vs sulfonylurea pre- and post-initiation when interpreting the findings of metformin on breast cancer incidence.

\section{ETHICS STATEMENT}

This study was approved by the Institutional Review Board expedited review at the University of North Carolina at Chapel Hill.

\section{FINANCIAL SUPPORT}

This study had no specific funding. The database infrastructure used for this study was funded by the Pharmacoepidemiology Gillings Innovation Lab (PEGIL) for the Population-Based Evaluation of Drug Benefits and Harms in Older US Adults (GIL
200811.0010), the Center for Pharmacoepidemiology, Department of Epidemiology, UNC Gillings School of Global Public Health; the CER Strategic Initiative of UNC's Clinical Translational Science Award (UL1TR001111); the Cecil G. Sheps Center for Health Services Research, UNC; and the UNC School of Medicine.

\section{REFERENCES}

1. Bodmer M, Meier C, Krahenbuhl S, et al. Long-term metformin use is associated with decreased risk of breast cancer. Diabetes Care 2010; 33: 1304-1308.

2. Chlebowski RT, McTiernan A, Wactawski-Wende J, et al. Diabetes, metformin, and breast cancer in postmenopausal women. Journal of clinical oncology : official journal of the American Society of Clinical Oncology 2012; 30: 2844-2852.

3. Ruiter R, Visser LE, van Herk-Sukel MP, et al. Lower risk of cancer in patients on metformin in comparison with those on sulfonylurea derivatives: results from a large population-based follow-up study. Diabetes Care 2012; 35: $119-124$.

4. van Staa TP, Patel D, Gallagher AM, et al. Glucose-lowering agents and the patterns of risk for cancer: a study with the General Practice Research Database and secondary care data. Diabetologia 2012; 55: 654-665.

5. Currie CJ, Poole CD, Gale EA. The influence of glucose-lowering therapies on cancer risk in type 2 diabetes. Diabetologia 2009; 52: 1766-1777.

6. Libby G, Donnelly LA, Donnan PT, et al. New users of metformin are at low risk of incident cancer: a cohort study among people with type 2 diabetes. Diabetes Care 2009; 32: 1620-1625.

7. Bosco JL, Antonsen S, Sorensen HT, et al. Metformin and incident breast cancer among diabetic women: a population-based case-control study in Denmark. Cancer epidemiology, biomarkers \& prevention : a publication of the American Association for Cancer Research, cosponsored by the American Society of Preventive Oncology 2011; 20: 101-111.

8. Redaniel MT, Jeffreys M, May MT, et al. Associations of type 2 diabetes and diabetes treatment with breast cancer risk and mortality: a population-based cohort study among British women. Cancer causes \& control : CCC 2012; 23: $1785-1795$.

9. Qiu H, Rhoads GG, Berlin JA, et al. Initial metformin or sulphonylurea exposure and cancer occurrence among patients with type 2 diabetes mellitus. Diabetes, Obesity \& Metabolism 2013; 15: 349-357.

10. Morden NE, Liu SK, Smith J, et al. Further exploration of the relationship between insulin glargine and incident cancer: a retrospective cohort study of older Medicare patients. Diabetes Care 2011; 34: 1965-1971.

11. Hong J-L, Josson Funk M, Buse J, et al. Comparative effect of Initiating Metformin versus Sulfonylureas on Breast Cancer Risk in Older Women. Epidemiology https://doi.org/10.1097/EDE.0000000000000635 [Epub ahead of print].

12. Suissa S, Azoulay L. Metformin and the risk of cancer: time-related biases in observational studies. Diabetes Care 2012; 35: 2665-2673.

13. Hong J-L, Jonsson Funk M, Lund J, et al. Differential Healthcare Utilization in Metformin Versus Sulfonylureas Users Pre-and Post-Initiation. Pharmacoepidemiology and Drug Safety 2014; 23: 64.

14. Fenton JJ, Zhu W, Balch S, et al. Distinguishing screening from diagnostic mammograms using Medicare claims data. Medical Care 2014; 52: e44-e51.

15. Fenton JJ, Onega T, Zhu W, et al. Validation of a Medicare Claims-based Algorithm for Identifying Breast Cancers Detected at Screening Mammography. Medical care 2013.

16. Sato T, Matsuyama Y. Marginal structural models as a tool for standardization. Epidemiology 2003; 14: 680-686.

17. Health, United States, 2014 - Women's Health: Preventive Care (Table 76). US Centers for Disease Control and Prevention, 2014. http://www.cdc.gov/nchs/ data/hus/hus14.pdf (Accessed February 12th, 2017).

18. Cronin KA, Miglioretti DL, Krapcho M, et al. Bias associated with self-report of prior screening mammography. Cancer epidemiology, biomarkers \& prevention : a publication of the American Association for Cancer Research, cosponsored by the American Society of Preventive Oncology 2009; 18: 1699-1705.

19. Rauscher GH, Johnson TP, Cho YI, et al. Accuracy of self-reported cancerscreening histories: a meta-analysis. Cancer epidemiology, biomarkers \& prevention : a publication of the American Association for Cancer Research, cosponsored by the American Society of Preventive Oncology 2008; 17: $748-757$.

20. Elkin EB, Ishill NM, Snow JG, et al. Geographic access and the use of screening mammography. Medical Care 2010; 48: 349-356. 
21. Breast Cancer: Screening. 2002. http://www.uspreventiveservicestaskforce.org/ Page/Document/UpdateSummaryFinal/breast-cancer-screening-2002 (Accessed February 12th, 2017)

22. Breast Cancer: Screening. 2009. http://www.uspreventiveservicestaskforce.org/ uspstf/uspsbrca.htm (Accessed February 12th, 2017).

23. Pace LE, He Y, Keating NL. Trends in mammography screening rates after publication of the 2009 US Preventive Services Task Force recommendations. Cancer 2013; 119: 2518-2523.

24. Molina Y, Thompson B, Ceballos RM. Physician and Family Recommendations to Obtain a Mammogram and Mammography Intentions: The Moderating Effects of Perceived Seriousness and Risk of Breast Cancer. Journal of women's health care $2014 ; 3$.

25. McCaul KD, Tulloch HE. Cancer screening decisions. Journal of the National Cancer Institute Monographs 1999; 52-8.

26. Schonberg MA, Marcantonio ER, Li D, et al. Breast cancer among the oldest old: tumor characteristics, treatment choices, and survival. Journal of clinical oncology : official journal of the American Society of Clinical Oncology 2010; 28: 2038-2045.

27. Cancer Rate (per 1,000 examinations) and Cancer Detection Rate (per 1,000 examinations) for 1,838,372 Screening Mammography Examinations from 2004 to 2008 by Age -- based on BCSC data through 2009. http:// breastscreening.cancer.gov/statistics/performance/screening/2009/rate_age.html (Accessed February 12th, 2017).

\section{SUPPORTING INFORMATION}

Additional Supporting Information may be found online in the supporting information tab for this article. 